\title{
Dynamic Response of Viscoelastic Rectangle Kirchhoff Plate
}

\author{
Zhendong Zhang ${ }^{1, a^{*}}$, Dawei Ma ${ }^{1, b}$, Qiang $\mathrm{He}^{1, \mathrm{c}}$ \\ ${ }^{1}$ School of Mechanical Engineering, Nanjing University of Science and Technology, Nanjing China \\ a zzd1157@163.com, b ma-dawei@ mail. njust. edu.cn, ${ }^{\mathrm{c}} 18260098162 @ 163 . c o m$
}

Keywords:Thermoviscoelastic constitutive equation, Temperature effect, Thin plate theory,Dynamic response

\begin{abstract}
On the basis of the thermoviscoelastic constitutive equation and classical Kirchhoff thin plate theory, the motion differential equation of viscoelastic rectangle plate is deduced. Then the expression of rectangle plate deflection under circle distributed dynamic load and temperature is deduced via Laplace integral transformation and triangular series methods. Results indicate that the deflection of plate increases gradually as the value of temperature increases. With the increase of time, the deflection enlarges too.
\end{abstract}

\section{Introduction}

Because of its low density and good mechanical properties, composite plate has been widely used in the automotive field, while these materials generally appear creep effect and stress relaxation property actually, it's necessary to consider the viscoelastic characteristic in the study of the dynamic response of the composite material plate.

An efficient numerical algorithm of the dynamic response of viscoelastic laminated plates composite plates under transient load was investigated by A. E. Assie[1] based on Mindlin-Reissner plate theory. Saeed Masoumi[2] used a multiscale method to analysis bending behavior of a laminated composite plate, while the differential quadrature method has been applied to investigate vibrations of viscoelastic thin plate with variable thickness by Yinfeng Zhou[3]. Raffaele Barretta[4] solved a viscoelastic equilibrium problems of isotropic viscoelastic functionally graded plate based on Kirchhoff plate theory in a closed form. Hong Liang Dai[5] based on von Karman nonlinear strain-displacement relationships and classical thin plate theory, then analyzed thermo-visco-elastic dynamic response of a viscoelastic composite thin narrow strip.

The purposes of this paper are to investigate the dynamic response of viscoelastic plate under circle distributed dynamic pressure and temperature. The governing equation of a visco-elastic plate is established based on the Kirchhoff thin-plate theory and thermoviscoelastic constitutive model.

\section{Constitutive equation}

The constitutive model of the thermoviscoelastic in the two-dimensional are presented as [5]:

$$
\left[\begin{array}{c}
\sigma_{x}(t) \\
\sigma_{y}(t) \\
\sigma_{x y}(t)
\end{array}\right]=\frac{1}{1-\mu^{2}}\left[\begin{array}{ccc}
E(t) & \mu E(t) & 0 \\
\mu E(t) & E(t) & 0 \\
0 & 0 & \frac{(1-\mu) E(t)}{2}
\end{array}\right] \otimes d\left[\begin{array}{c}
\varepsilon_{x}-\alpha \Delta T(t) \\
\varepsilon_{y}-\alpha \Delta T(t) \\
\gamma_{x y}
\end{array}\right]
$$

Where $E(t), \mu, \alpha$ and $T(t)$ are stress relaxation modulus, Poisson's ratios, linear expansion coefficient and plate temperature field on the thin plate respectively.

\section{Stress relaxation function}

The Burgers model, which is shown in Fig.1, can reflect mechanical properties of viscoelastic materials well. 


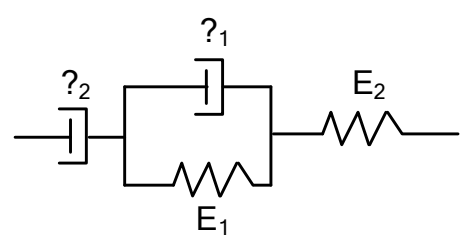

Fig.1 Burgers model

The stress relaxation function $E(t)$ can be written as:

$$
E(t)=\frac{E_{1}}{\alpha-\beta}\left[\left(\frac{\eta_{2}}{E_{2}}-\beta\right) e^{-\beta t}-\left(\frac{\eta_{2}}{E_{2}}-\alpha\right) e^{-\alpha t}\right]
$$

Where $\alpha=\frac{p_{1}+\sqrt{p_{1}^{2}-4 p_{2}}}{2 p_{2}}, \quad \beta=\frac{p_{1}-\sqrt{p_{1}^{2}-4 p_{2}}}{2 p_{2}}, \quad p_{1}=\frac{\eta_{1}}{E_{1}}+\frac{\eta_{1}+\eta_{2}}{E_{2}}, \quad p_{2}=\frac{\eta_{1} \eta_{2}}{E_{1} E_{2}}$.

\section{The dynamic equilibrium equations of the plate}

A Kirchhoff plate model under circle distributed dynamic load is shown in Fig.2, where a, b, h denote the width, the length and the thickness respectively.

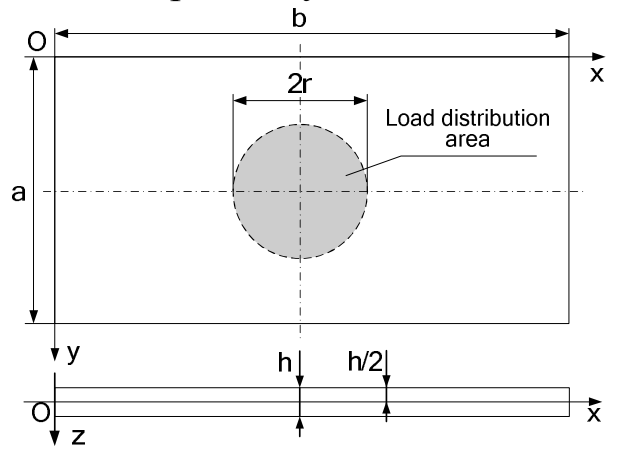

Fig.2 Rectangle plate model

According to the Kirchhoff thin plate theory, strain components can be written as:

$$
\left\{\varepsilon_{x}=-z \frac{\partial^{2} w}{\partial x^{2}}, \quad \varepsilon_{y}=-z \frac{\partial^{2} w}{\partial y^{2}}, \quad \gamma_{x y}=-2 z \frac{\partial^{2} w}{\partial x \partial y}\right.
$$

Moment equilibrium equation is given as

$$
\frac{\partial^{2} M_{x}(x, y, t)}{\partial x^{2}}+2 \frac{\partial^{2} M_{x y}(x, y, t)}{\partial x \partial y}+\frac{\partial^{2} M_{y}(x, y, t)}{\partial y^{2}}+F(x, y, t)-m_{b} \frac{\partial^{2} W(x, y, t)}{\partial t^{2}}=0
$$

Where $F(x, y, t)$ is pressure, $W(x, y, t)$ is deflection of plate. Internal forces of plate $M_{x}, M_{y}, M_{x y}$ and unit area density $m_{b}$ are defined as:

$$
\left[M_{x}, M_{y}, M_{x y}\right]=\int_{-h / 2}^{h / 2}\left[\sigma_{x}, \sigma_{y}, \sigma_{x y}\right] z d z, \quad m_{b}=\rho h
$$

Where $\rho, h$ are, respectively, the density of plate and plate thickness.

Apply the Laplace integral transformation on Eq. (3), Eq. (1) and substitute them into the Laplace integral transformation of Eq. (4), then we have

$$
\left(\frac{\partial^{4} \bar{W}}{\partial x^{4}}+2 \frac{\partial^{4} \bar{W}}{\partial x^{2} \partial y^{2}}+\frac{\partial^{4} \bar{W}}{\partial y^{4}}\right) \int_{-h / 2}^{h / 2} \frac{\bar{E}(z, s)}{1-\mu^{2}} z^{2} d z+s^{2} m_{b} \bar{W}=\bar{F}
$$

Where $\bar{E}(z, s)$ is the Laplace integral transformation of stress relaxation modulus. 


\section{Solution of equilibrium equations}

The boundary conditions for simply supported on four edges can be written as:

$$
\left.\begin{array}{ll}
W(x, y, t)=\frac{\partial^{2} W(x, y, t)}{\partial x^{2}}=0 & x=0, a \\
W(x, y, t)=\frac{\partial^{2} W(x, y, t)}{\partial y^{2}}=0 & y=0, b
\end{array}\right\}
$$

Circle distributed dynamic pressure $F(x, y, t)$ is defined as:

$$
F(x, y, t)=f(t) \times H\left\{r^{2}-\left[\left(x-x_{0}\right)^{2}+\left(y-y_{0}\right)^{2}\right]\right\}
$$

Where $f(t)=0.1 \sin (4 \pi t), r,\left(x_{0}, y_{0}\right)$ and $H(x, y)$ are, respectively, the average load intensity, radius of load distribution area, the center coordinates and Heaviside function.

The triangular-series-form displacement functions which satisfy the boundary conditions are defined as:

$$
W(x, y, t)=\sum_{l=1}^{\infty} \sum_{m=1}^{\infty} q_{l m}(t) \cdot \sin \left(\alpha_{l} x\right) \cdot \sin \left(\beta_{m} y\right)
$$

Where $q_{l m}(t)$ is expansion coefficient, $\alpha_{l}=\frac{l \pi}{a}, \beta_{m}=\frac{m \pi}{b}$.

Similarly, $F(x, y, t)$ can be written as:

$$
F(x, y, t)=\sum_{l=1}^{\infty} \sum_{m=1}^{\infty} f_{l m}(t) \cdot \sin \left(\alpha_{l} x\right) \cdot \sin \left(\beta_{m} y\right)
$$

Where

$$
f_{l m}(x, y, t)=\frac{4}{a b} \int_{0}^{a} \int_{0}^{b} F(x, y, t) \cdot \sin \left(\alpha_{l} x\right) \cdot \sin \left(\beta_{m} y\right) d x d y
$$

Substituting Eq. (7) into upper equation, then

$$
f_{l m}(x, y, t)=\frac{4 f(t)}{a b} \cdot G_{l m}
$$

Where

$$
G_{l m}=\iint_{A} \sin \left(\alpha_{l} x\right) \cdot \sin \left(\beta_{m} y\right) d x d y
$$

Where A is closed area of load surface.

Substituting the Laplace integral transformation of Eq.(8) and Eq.(9) into Eq.(5), then we have

$$
\bar{q}_{l m}(s)=\frac{4 G_{l m} \bar{f}(s)}{a b\left[\left(\alpha_{l}^{2}+\beta_{m}{ }^{2}\right)^{2} \int_{-h / 2}^{h / 2} \frac{\bar{E}(z, s)}{1-\mu^{2}} z^{2} d z+s^{2} m_{b}\right]}
$$

Transforming Eq.(5) into Laplace integral form and substituting it into Eq.(8), then the transverse displacement of plate can be written as:

$$
W(x, y, t)=\sum_{l=1}^{\infty} \sum_{m=1}^{\infty}\left\{\frac{4 \sin \left(\alpha_{l} x\right) \sin \left(\beta_{m} y\right) G_{l m}}{a b\left(\alpha_{l}{ }^{2}+\beta_{m}{ }^{2}\right)^{2}} \times \mathbf{L}^{-1}\left[\bar{f}(s) /\left(\int_{-h / 2}^{h / 2} \frac{\bar{E}(z, s)}{1-\mu^{2}} z^{2} d z+s^{2} m_{b}\right)\right]\right\}
$$




\section{Numerical results and discussions}

The values of useful parameters are presented in Tab.1, Tab.2 gives the parameter value of Burgers model.

Tab.1 The values of useful parameters

\begin{tabular}{ccc}
\hline Parameters & Unit & Value \\
\hline$\rho$ & $\mathrm{Kg} / \mathrm{m}^{3}$ & 2200 \\
$h$ & $\mathrm{~m}$ & 0.02 \\
$\mu$ & - & 0.25 \\
$\mathrm{a}, \mathrm{b}$ & $\mathrm{m}$ & $0.5,0.5$ \\
$\left(x_{0}, \mathrm{y}_{0}\right)$ & $\mathrm{m}$ & $(0.25,0.25)$ \\
$r_{0}$ & $\mathrm{~m}$ & 0.1
\end{tabular}

Tab.2 The parameter value of Burgers model

\begin{tabular}{ccccc}
\hline $\begin{array}{c}\text { Temperature } \\
\left({ }^{\circ} \mathrm{C}\right)\end{array}$ & $E_{1}(\mathrm{~Pa})$ & $\eta_{1}(\mathrm{~Pa} \cdot \mathrm{s})$ & $E_{2}(\mathrm{~Pa})$ & $\eta_{2}(\mathrm{~Pa} \cdot \mathrm{s})$ \\
\hline 20 & $4.0 \times 10^{8}$ & $32708601.30 \times 10^{8}$ & 74860 \\
\hline 35 & $3.0 \times 10^{8}$ & $16415300.58 \times 10^{8}$ & 111360 \\
50 & $1.2 \times 10^{8}$ & $15597500.60 \times 10^{8}$ & 54980 \\
60 & $1.0 \times 10^{8}$ & $16846500.45 \times 10^{8}$ & 34600 \\
\hline
\end{tabular}

Fig. 3 gives the deflection of the plate with different temperatures at circle distributed dynamic load. The temperature parameters are set as $20^{\circ} \mathrm{C} 、 35^{\circ} \mathrm{C} 、 50^{\circ} \mathrm{C}$ and $60^{\circ} \mathrm{C}$ respectively.

The outcomes of the Fig. 3 can be summarized as: (1) The higher the temperature, the greater the deflection; (2) Due to the creep characteristics, the peak value of plate deflection increase with the passing of time.

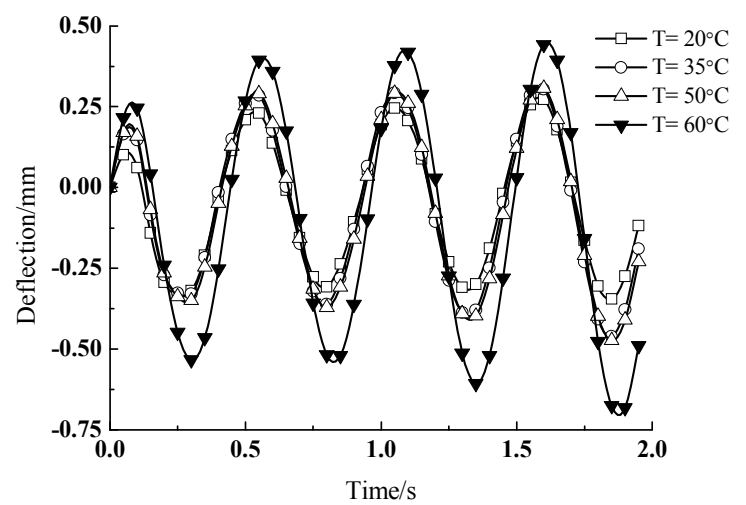

Fig.3 The deflections of plate at different temperatures

\section{Conclusions}

The presented paper establish governing equation of a visco-elastic plate based on the Kirchhoff plate theory and two-dimensional thermo-visco-elastic constitutive relation, then the dynamic response of plate is analyzed. The results show that the deflection is obvious as the temperature increases at the same harmonic excitation load.

\section{References}

[1] A. E. Assie, M. A. Eltaher, F. F. Mahmoud, Journal of Mechanical Science and Technology, 25(5) (2011) 1129-1140.

[2] Saeed Masoumi, Manouchehr Salehi, Mehdi Akhlaghi, Acta Mechanica sinica, 223 (2012) 2459-2476.

[3] Yinfeng Zhou, Minwang Zhong, Meccanica, 49 (2014) 2817-2828.

[4] Raffaele Barretta, Raimondo Luciano, Composite Structures, 118 (2014) 448-454.

[5] Liangdai Hong, Liqi Li, Boliu Hai, Journal of Mechanical Science and Technology, 29(2) (2015) 625-636. 